\title{
HETEROGENEOUS COMMUNICATION IN BETWEEN TWO WIRELESS TECHNIQUES: BLUETOOTH AND WI-FI
}

\author{
SOJWAL A. PATIL
}

Department of E\&TC Engg., SKNCOE, Pune, India

\section{ABSTRACT}

Bluetooth (802.15.1), ZigBee (802.15.4) and Wi-Fi

(802.11) are the wireless protocols used for wireless communication. Bluetooth and ZigBee consumes less power and works on short range communication whereas Wi-Fi consumes high power and is suitable for long range. Hence heterogeneous communication between low power consumption wireless techniques with more efficient wireless technique is needed to be developed. The proposed system introduces a method which creates compatibility and has unique feature of establishing communication between Bluetooth and Wi-Fi by which data transmission over long distance can takes place. Also the data on internet can be received by monitoring the object condition continuously. The mentioned system is an initiative which establishes interoperability between Bluetooth and Wi-Fi.

KEYWORDS: Wireless Communication, Bluetooth, ZigBee, Wi-Fi.

Received: Mar 05, 2018; Accepted: Apr 25, 2018; Published: Jun 14, 2018; Paper Id.: IJCSEITRJUN201813

\section{INTRODUCTION}

ZigBee nodes in the wireless sensor network communicate with each other. Applications where low data transfer is needed like monitoring and controlling, ZigBee network is useful but applications where larger data transfer is required, this network is not suitable. Now-a-days devices are manufactured having Bluetooth bee and Wi-Fi bee. All of these uses a protocol which establishes communication between Bluetooth and ZigBee or Wi-Fi and ZigBee respectively [1][2]. When higher bit rate is required, these nodes fail to send data [3]. The solution to this problem can be a system which directly communicates with Bluetooth and Wi-Fi by using their respective stack chips. A processor is required in both Bluetooth bee [1], Wi-Fi bee [2] to set up a link so as the proposed system. Bluetooth module has less on chip ROM which fails to mount the compatibility protocol, hence needs a host processor for proper functioning.

In [3] Artur Baniukevic proposed hybrid approach to achieve better indoor positioning. Wi-fi, RFID or Bluetooth can be used for the same. Technologies using Wi-fi could be easy ro deploy but lacks in accuracy. Whereas, RFID or Bluetooth needs considerable investements to achieve good accuracy. So Artur Baniukevic worked on hybrid approach in which available Wi-fi infrastructure is used to introduce hotspot to improve positioning.

Jin-Shyan Lee et al. [4] gives the features and behavior of Bluetooth, UWB, ZigBee and Wi-Fi in terms of various metrics such as transmission time, data codig efficiency, power consumption and complexity. It is found that UWB and Wi-Fi consumes more power for transmission and reception as compared to other two protocols i.e. Bluetooth and Zigbee but the same time requires less energy. 
The four wireless protocols works on different ranges. IEEE have standardize them as 802.15.1, 802.15.3, 802.15.4 and 802.11 a/b/g respectively. The PHY and MAC layer allows wireless communication over range of 10-100 m. In [5] Ferro and Portoti, comparison of Bluetooth and Wi-Fi over capacity, security, network topology, power consumption and quality of service support is done.[6] have compared MAC of IEEE 802.11e and 802.15.3 in throughput. Both the protocols differ in very small values. Also 802.15.3 has easier power management than the other i.e. 802.11e. Whereas Baker [7] found that, in between Zigbee and Bluetooth, Zigbee meets the real industrial needs as it has longer battery operation, greater useful range, flexibility in number of dimensions.

The proposed system helps to reduce the use of Wi-Fi in many hand held devices so reduces the power consumption as Bluetooth and Wi-Fi connectivity will be there. Immediate access to information and ease of connectivity acts as motivation for this concept.

This paper presents the design and the implementation of a communication system between Bluetooth and Wi-Fi. A sensor is used to demonstrate the communication which monitors the temperature of any device and then sends it to WiFi by using proposed system. The system along with any sensor can further be used to collect the data regarding any object. Because of its sensors connected system, it will provide authorities with more information and control in to identify and fix these problems. At the user end, a user would know when devices needed replacing, repairing or recalling. This will allow them to operate more profitably, giving extra incentives.

The Stack is responsible for establishing communication between Bluetooth and Wi-Fi, so some changes have to be made in it. The new stack with both the technology has to be developed, to do so the access to the core of stack is essential. Currently, as Bluetooth and Wi-Fi stacks are patented, the availability of internal design of stack chip of both the technologies is difficult. So to solve this challenge, other approach is discussed and implemented in this paper.

The proposed system helps Bluetooth enabled devices to send data over longer ranges and to connect to internet very easily using Wi-Fi. Mainly, the system contributes to establish communication link between Bluetooth and Wi-Fi. Under IOT concept representation of device on internet is suggested. Any device in this system can deploy the collected data on internet using a strong wireless system which has efficient protocols to communicate with Internet. Bluetooth in responsible for collecting the data and Wi-Fi deploys it on internet which makes input as Bluetooth and output as Wi-Fi.

The rest of the paper is organized as follows. Section II provides the necessary background. A detailed description of each \& every block along with their design which is represented in Section III. Then the focus is set on the novel system adopted by the proposed system to address the interaction between the two wireless technologies. Section IV describes about the software requirement along with flow of the system operation. Section V and VI represents result, advantages, limitations and applications respectively. Finally, Section VII is concluded with the future work

\section{BLUETOOTH AND WI-FI MODULE}

The given system will be using Aubtm 20 Bluetooth module and RN-131g Wi-Fi module for transceiving the information over Bluetooth and Wi-Fi.

Aubtm 20 supports Bluetooth core V1.2, with a data speed of approximately $720 \mathrm{kbps}$, RN 131g Wi-Fi module is 2.4GHz IEEE $802.11 \mathrm{~b} / \mathrm{g}$ transceiver which supports data speed of 1Mbps. The below scenario explains the conversion of Bluetooth to Wi-Fi communication. Hand held devices such as mobiles or other devices having Bluetooth connectivity will 
send the data to Bluetooth and then it will be sent to Wi-Fi converter. The converter will convert the Bluetooth data into Wi-Fi data and it can be sent over Wi-Fi range respectively Wi-Fi receiver Bluetooth to Wi-Fi converter and the compatibility protocol is implemented in processors which will establish the communication between them.[1]

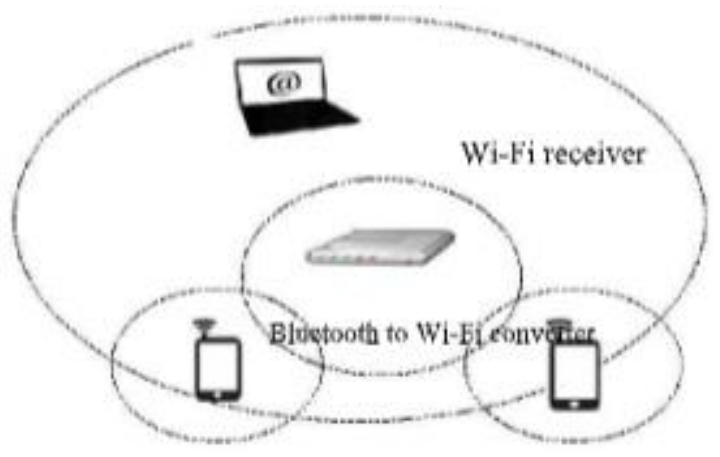

Figure 1: General Concept

\section{COMPARISON OF DIFFERENT SYSTEMS WITH PROPOSED SYSTEM}

1. Libelium , a technology leader in wireless sensor networks, announces the launch new Platform [9]. The system is a combination of Bluetooth stack chip and Zigbee stack chip. The device like Bluetooth Bee and Wi-Fi Bee has a combination of Bluetooth, Zigbee and Wi-Fi and Zigbee respectively and the compatibility protocol is implemented in processors which will establish the communication between them.[1]

2. Wi-Fi Bee is a ZigBee Pro socket compatible standalone node useful for Wireless Sensor Network. It integrates Microchip IEEE 802.11 Wi-Fi transceiver module MRF24WB0MA and an 8bit AVR MCU ATMega328P. [2]

3. Meshlium X treme is router developed by libelium. Meshlium may communicate with ZigBee, Wi-Fi and Bluetooth sensor nodes and then send the information collected to the Cloud by either $3 \mathrm{G}$ or Ethernet interfaces depending on the communication available at any time. It is not converting the data into other format but it is a multiprotocol router which can access the data through any 3 mentioned wireless protocols and then it can deploy it on cloud through $3 \mathrm{G}$ or Ethernet. It has 4 antennas which are able to sense Bluetooth, Wi-Fi and Zigbee separately. The router is not able to convert the data which is received by Bluetooth into Wi-Fi.[11]

4. The system described here will be a combination of Bluetooth and Wi-Fi and the compatibility protocol can be implemented in ARM processor. The working is described below.

\section{WORKING OF PROPOSED SYSTEM}

It consists of Bluetooth receiver which will receive the information sent by the sensor node through Bluetooth. Sending the information requires specific functions. The Bluetooth module will go in command mode to accept the commands as required. Then it will go in sniff mode, sniffing is a process of listening to specific types of commands that occur periodically. Sniffing is used for devices that must continuously be in contact with the master.

The Bluetooth sniff mode is used to reduce the power consumption of the device as the receiver can be put into standby between sniff cycles. To set the device, set function will be evoked and module will be set. To have its own identity, name will be given to the Bluetooth module. The security settings will be done after that.

The security and service setting has to be checked. In connection establishment Phase, the enquiry has to be done 
to connect to specific device. In configuration phase the address and signal strength need to take into consideration. In data transmission phase the data will get transferred to the device. The host processor will then take the data and by setting IP address and other settings of Wi-Fi will pass this information to Wi-Fi transmitter.

We have to connect this Wi-Fi transmitter to Wi-Fi receiver so that the data can be sent on the computer. A device that can use Wi-Fi such as a personal computer, video game console, smart phone, tablet, or digital audio player can connect to a Wi-Fi network via a wireless network access point. Such an access point or hotspot has a range of about 20 meters 65 feet indoors and a greater range outdoors. Hotspot coverage can comprise an area as small as a single room with walls that block radio waves or as large as many square miles this is achieved by using multiple overlapping access points.

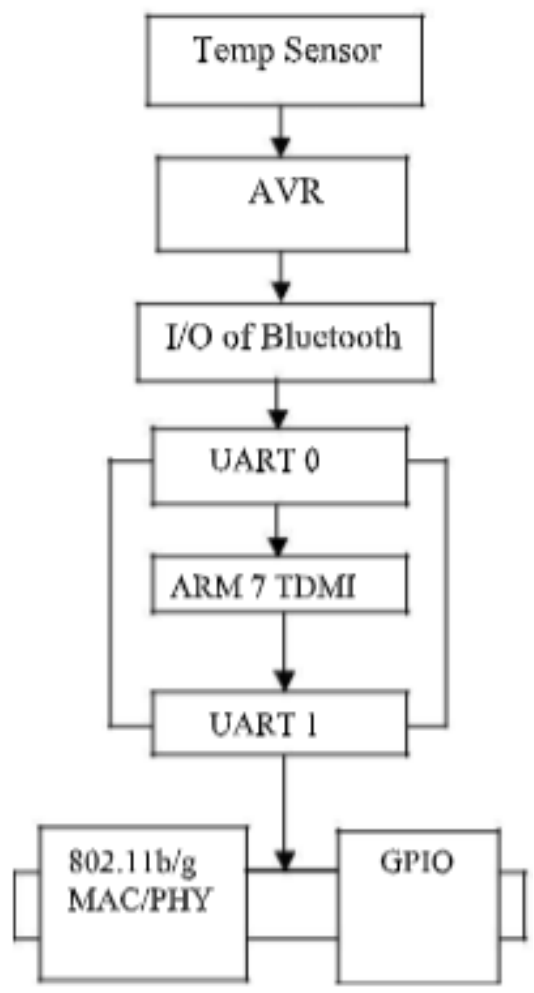

Figure 2: System Operation

\section{RESULTS AND DISCUSSIONS}

The proposed system consists of sensor node which is connected to the Bluetooth module. Sensor measures the temperature of any system and displays it on LCD. This Bluetooth module will transmit the data to Wi-Fi module. At I/P side of this module Bluetooth receiver is mounted and at output side Wi-Fi is connected, on this module another LCD is connected, so Bluetooth module and Wi-Fi node LCD both should display. 


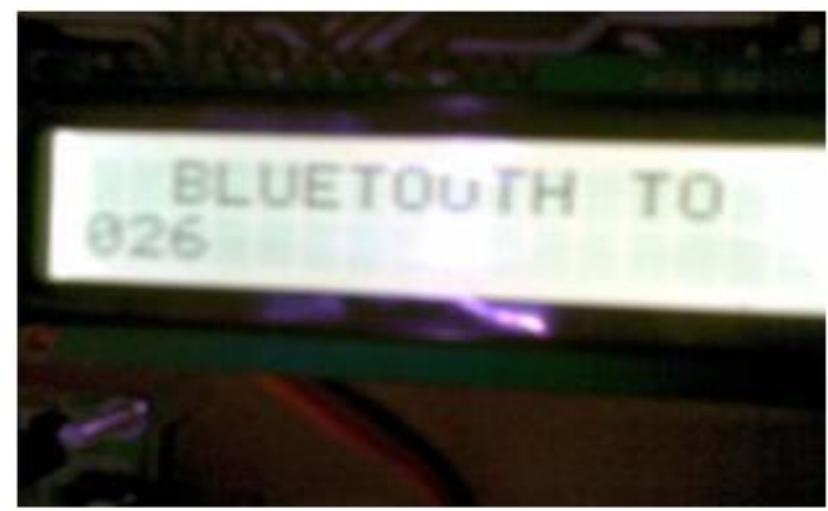

Figure 3: Display on Bluetooth Side

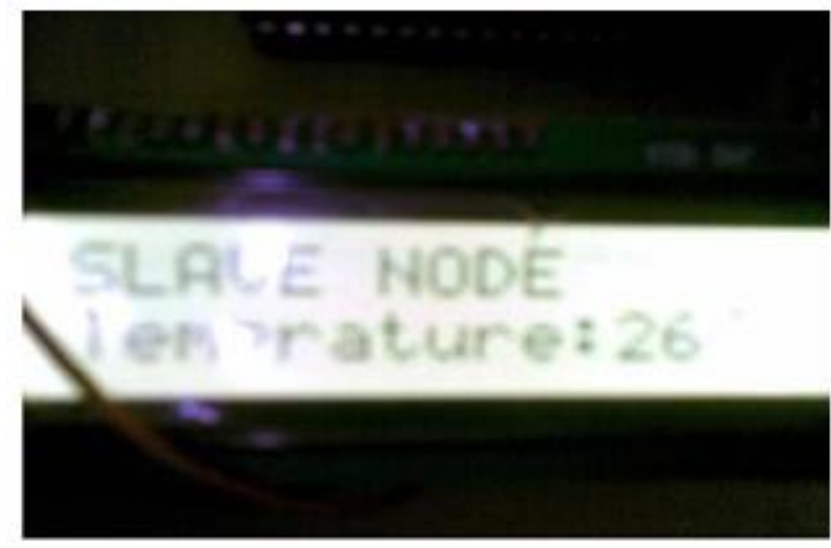

Figure 4: Display on Wi-Fi Node

This is the verification of Bluetooth to Bluetooth communication. Next we will see the verification of Bluetooth to Wi-Fi communication There are no significant interferences of Bluetooth on Wi-Fi and vice versa [1].

Wi-Fi has compatible protocols for internet than Bluetooth. We are sending 40 bit data through this system.To verify whether we are getting the data through $\mathrm{Wi}$ - Fi, we need a display on the receiver section of Wi-Fi, so we will be using hyper terminal of a computer.

The transmitted data by Wi-Fi module will get received by the Wi-Fi receiver of Inspiron 14z. In order to receive it, we need to search and connect the Wi-Fi module to Inspiron $14 \mathrm{z}$ and by connecting it to hyper terminal, we can verify the data.

Table I: Module and its Result

\begin{tabular}{|c|l|l|}
\hline No. & \multicolumn{1}{|c|}{ Module } & \multicolumn{1}{c|}{ Result } \\
\hline 1 & $\begin{array}{l}\text { Sensor node and } \\
\text { Bluetooth Transmitter }\end{array}$ & $\begin{array}{l}\text { Transmits temperature and } \\
\text { display it on LCD }\end{array}$ \\
\hline 2 & $\begin{array}{l}\text { Bluetooth receiver } \\
\text { and Wi-Fi Transmitter }\end{array}$ & $\begin{array}{l}\text { Receiver receives the data and } \\
\text { shows it on LCD whether } \\
\text { same data is received or not } \\
\text { and transmits through Wi-Fi }\end{array}$ \\
\hline 3 & $\begin{array}{l}\text { Wi-Fi transmitter and } \\
\text { Wi-Fi Receiver }\end{array}$ & $\begin{array}{l}\text { On HyperTerminal the same } \\
\text { data gets Displayed }\end{array}$ \\
\hline
\end{tabular}

With regards to power consumption, Bluetooth and Zigbee consume less power and works for short range. For 
long ranges, $\mathrm{Wi}-\mathrm{Fi}$ is used, which consumes more power compared to the other two protocols i.e. Bluetooth and Zigbee. Figure 5 below shows the comparison of Bluetooth, Zigbee and Wi-Fi based on its power consumption in $\mathrm{mW}$. Wi-Fi consumes more power than other two as it works for longer range

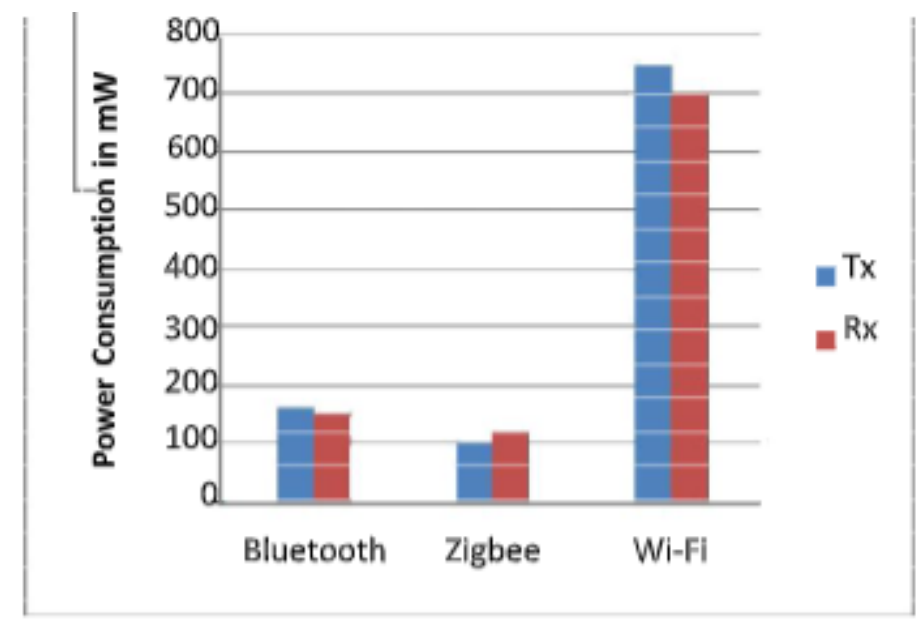

Figure 5: Comparison of Power Consumption for Each Protocol

Figure 6 compares the energy consumption based on bit rate. Better efficiency in energy consumption is achieved by Wi-Fi.

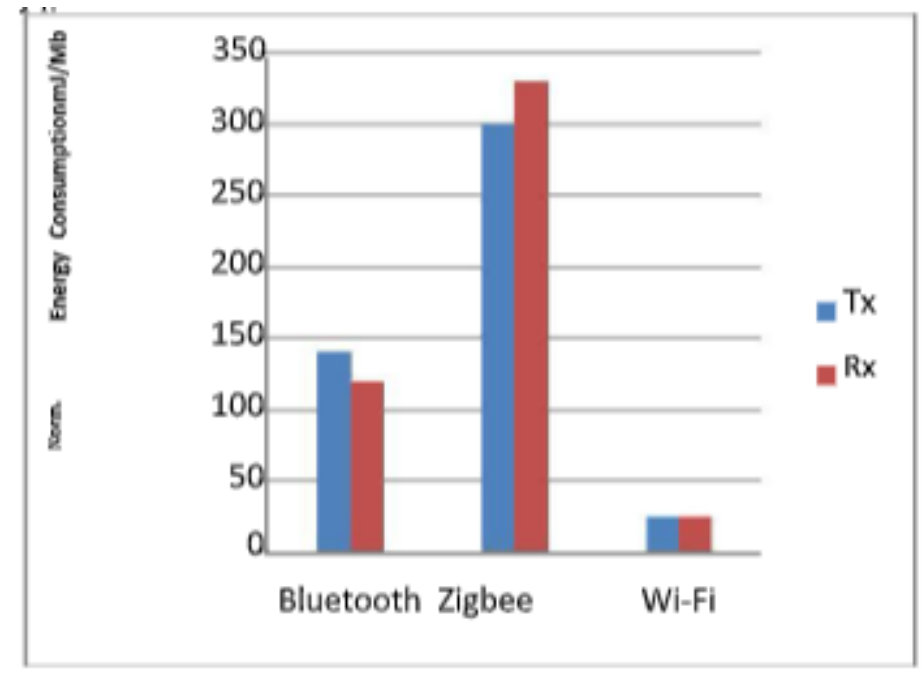

Figure 6: Comparison of the Normalized Energy Consumption for Each Protocol

Figure 7 shows system performance. As shown in graph the data sent by Bluetooth will be received by Wi-Fi nearly 120 bps. As distance will increase the speed of the communication between Bluetooth to Wi-Fi will decrease till 100 feet. The system requires 8.33 milliseconds to transfer one bit. The Wi-Fi to Bluetooth curve is drawn by keeping distance constant between two Bluetooth to Bluetooth node, after 100 feet the connection will get terminated. The proposed system is using Aubtm 20 Bluetooth module and RN131g Wi-Fi module. Wi-Fi module supports 802.11 b/g standards. Bluetooth to Bluetooth speed is ideally $720 \mathrm{kbps}$ for v1.2 and practically it gives the speed of 300 bps till 3 feet and will get terminated after that.

Brown line signifies Bluetooth to Bluetooth and blue line details Wi-Fi to Bluetooth (Bluetooth to Bluetooth distance is 3 feet). $\mathrm{Y}$-axis signifies the speed in bps. 


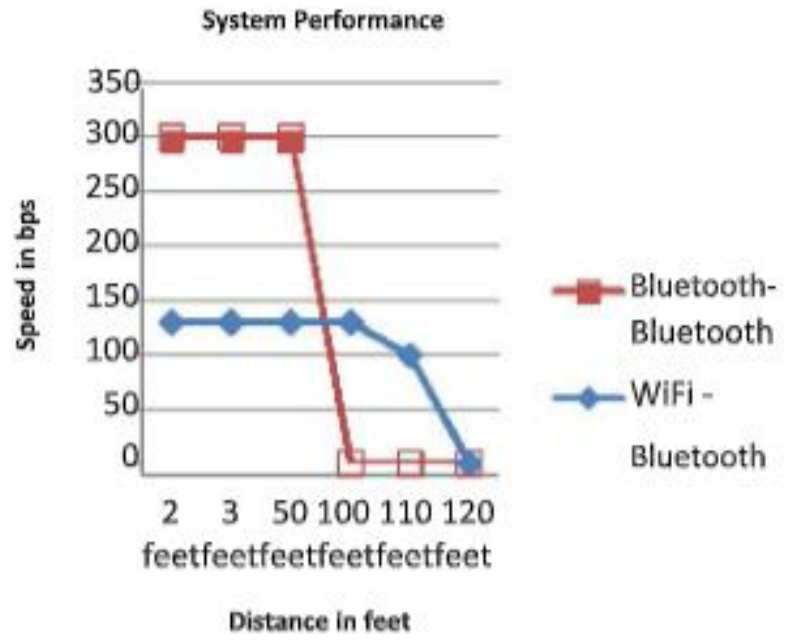

Figure 7: Result Analysis

\section{ADVANTAGES, LIMITATIONS AND APPLICATIONS}

\section{A. Advantages}

- For collecting the data, system uses Bluetooth which has higher data rate than Zig-Bee so in a case of sensor network Zig-Bee can be replaced by this Bluetooth.

- Smart phones have Bluetooth as well as Wi-Fi transceiver; proposed system helps to reduce the design of Smartphone by eliminating the need of Wi-Fi in Smartphone or any other handheld device as the system can convert the data into Wi-Fi compatible format.

- By using proposed circuit as one node in established Bluetooth-based sensor network, a user can send the data over longer range with high data rate.

Depending upon application the system can use other high-speed host processor.

\section{Limitations}

- The range of Wi-Fi should be available. But this problem can be solved by using Wi-Fi direct.

- Synchronization is necessary.

\section{Applications}

- Moving Consumer can place the order in his Bluetooth enabled device and it can be deployed on the internet hence their need can be fulfilled.

- It has number of applications under Internet of Things. The Bluetooth sensor node can be attached to any device and can virtually be represented on the internet.

- Remote patient monitoring.

- Industrial Control or Automation.

- Health monitoring and Sport / Fitness. 


\section{CONCLUSION AND FUTURE SCOPE}

Immediate access to information and ease of connectivity are the key drivers for Wi-Fi and Bluetooth becoming the communications protocol standards world-wide. The system can be modified for other host processor to ease application development processes. As a result, proposed system module is ideal for original equipment manufacturers wanting to quickly add $\mathrm{Wi}-\mathrm{Fi}$ and Bluetooth connectivity within a broad range of products for the world-wide marketplace. The implemented system successfully established the interoperability between Bluetooth and Wi-Fi system.

In future, the module could be designed on a single chip so that it can receive any kind of data that is Bluetooth or Wi-Fi and can convert into other. Also could provide a solution for high performance by using high gain antennas and amplifiers so that system can send and receive the data more efficiently. The module can be design to fit into small spaces.

\section{REFERENCES}

1. www.seeedstudio.com/wiki/Bluetooth_Bee

2. www.seeedstudio.com/wiki/WI-FI_Bee

3. Artur Baniukevic, Dovydas Sabonis, Christian S. Jensen, Hua Lu "Improving Wi-Fi Based Indoor Positioning Using Bluetooth Add-Ons, "IEEE International Conference on Mobile Data Management 2011

4. Jin-Shyan Lee, Yu-Wei Su, and Chung-Chou Shen "A Comparative Study of Wireless Protocols: Bluetooth, UWB,

5. ZigBee, and Wi-Fi," Information \& Communications Research Labs

6. E. Ferro and F. Potorti, "Bluetooth and Wi-Fi wireless protocols: A survey and a comparison," IEEE Wireless Commun., vol. 12, no. 1, pp.12-16, Feb. 2005.

7. Baker, N. "ZigBee and Bluetooth: Strengths and weaknesses for industrial applications," IEE Computing \& Control Engineering, vol. 16, no. 2, pp 20-25, April/May 2005.

8. $\quad$ Blue core 4 Rom Product Data Sheet

9. User manual educational practice board for LPC $2138 / 48$

10. The Insider,s guide to the Philips ARM 7 an engineer's introduction to LPCxxxx series. [10]http://www.neondrum.com/public/public_release.php?id= 970

11. Microchip product discription Industrial Technology Research Institute (ITRI) Hsinchu,Taiwan jinshyan_lee@itri.org.tw

12. http://www.libelium.com/products/meshlium/

13. D. Porcino and W. Hirt, "Ultra-wideband radio technology: Potential and challenges ahead," IEEE Commun. Mag., vol. 41, no. 7, pp. 66-74, July 2003.

14. J. S. Lee, "Performance evaluation of IEEE 802.15.4 for low-rate wireless personal area networks," IEEE Trans. Consumer Electron., vol. 52, no. 3, pp. 742-749, Aug. 2006.

15. J. S. Lee and Y. C. Huang, "ITRI ZBnode: A ZigBee/IEEE platform for wireless sensor networks," in Proc. IEEE Int. Conf Systems, Man \& Cybernetics, Taipei, Taiwan, Oct. 2006, pp. 1462-1467.

16. A. Sikora and V. F. Groza, "Coexistence of IEEE802.15.4 with other systems in the 2.4 GHz-ISM-Band," in Proc. IEEE Instrumentation \& Measurement Technology Conference, Ottawa, May 2005, pp.1786-1791.

17. K. Shuaib, M. Boulmalf, F. Sallabi, and A. Lakas, "Co-existence of Zigbee and WLAN: A performance study," in Proc. 
IEEEIIFIP Int. Conf Wireless \& Optical Communications Networks, Bangalore, India, April 2006.

18. P. S. Neelakanta and H. Dighe, "Robust factory wireless communications: A performance appraisal of the Bluetooth and the ZigBee collocated on an industrial floor," in Proc. IEEE Int. Conf Ind. Electron. (IECON'03), Roanoke, VA, Nov. 2003, pp. 2381-2386.

19. Cambridge Silicon Radio, BlueCore2-External Product Data Sheet.Cambridge, UK, Aug. 2006.

20. Freescale, XS11O UWB Solution for Media-Rich Wireless Applications.vSan Diego, CA, Dec. 2004.

21. Chipcon, CC2430 Preliminary Data Sheet (rev. 1.03). Oslo, Norway, 2006.

22. Conexant, Single-Chip WLAN Radio CX53111. Newport Beach, CA,2006. 
